\title{
Monoglucosyl rutin facilitates recovery from eyestrain in visual display terminal users: a randomized, placebo-controlled, double-blind, crossover study
}

Hiroshi Ashigai ( $\square$ Hiroshi_Ashigai@kirin.co.jp )

Research Laboratories for Health Science and Food Technologies, Kirin Co. https://orcid.org/00000002-1204-2502

\section{Tomoka Kanaya}

Research Laboratories for Health Science and Food Technologies, Kirin Co.

Hitomi Fujii

Research Laboratories for Health Science and Food Technologies, Kirin Co.

\section{Yasuji Kawachi}

Research Laboratories for Health Science and Food Technologies, Kirin Co.

\section{Masayoshi Kajita}

Kajita Eye Clinic

\section{Research}

Keywords: Monoglucosyl rutin, Eyestrain, Visual display terminal, Clinical study

Posted Date: December 30th, 2019

DOI: https://doi.org/10.21203/rs.2.19584/v1

License: (c) (i) This work is licensed under a Creative Commons Attribution 4.0 International License.

Read Full License 


\section{Abstract}

\section{Background}

Eyestrain is a serious quality of life problem, especially in the workplace. In particular, work at visual display terminals (VDTs) is a common cause of eyestrain. Currently, there are many workers who work at VDTs and tend to feel eyestrain. Rutin, derived from various food plants such as the Japanese pagoda tree (Styphnolobium japonicum), is a known polyphenol compound. Monoglucosyl rutin (MGR) is a highly water-soluble a-glucose adduct of rutin. Previous studies have demonstrated that rutin improved dry eye and also affected the microvascular blood flow in animal experiments. Based on these findings, we hypothesized that MGR could alleviate eyestrain caused by work at VDTs. In this clinical study, we evaluated whether MGR could improve eyestrain in healthy adults.

\section{Methods}

This study was designed as a randomized, placebo-controlled, double-blind, crossover study. Twenty healthy Japanese adults who tended to feel eyestrain were recruited to participate. During the first test period, the participants were asked to drink either a test food containing $377 \mathrm{mg}$ of MGR or a placebo food without MGR. After the food intake, the participants did personal computer work at VDT for $30 \mathrm{~min}$ and then relaxed for $20 \mathrm{~min}$. We evaluated the participants' eyestrain using an objective parameter (highfrequency component 1, HFC1) and subjective parameters (visual analog scale). After 7 days, for the second test period, the participants consumed an alternate food following the same protocol as during the first test period.

\section{Results}

The objective parameter of eyestrain (HFC1) was significantly lower in the MGR group than that in the placebo group. The subjective parameters of eyestrain and waist stiffness were improved in the MGR group in comparison with those in the placebo group.

\section{Conclusions}

MGR supplementation has the potential to objectively and subjectively improve eyestrain symptoms due to VDT use.

\section{Background}

Currently, many people use computing devices with visual display terminals (VDTs), not only for business but also for private reasons. In particular, the number of smartphone users constantly grows worldwide. The increasing use of computers and their related output devices causes a clinical condition called computer vision syndrome [1]. Computer vision syndrome includes extraocular symptoms, which involve the musculoskeletal system, peripheral nervous system, and skin. Although the VDT use is associated with a large number of extraocular symptoms, eye symptoms are the most frequent complaints among 
users. Using VDTs involves a prolonged near-vision task, but the symptoms associated with their use, such as blur, dryness, and eyestrain, are significantly more severe than those associated with similar tasks without using VDTs. VDT use causes eyestrain, stiffness of the shoulder and neck, and therefore, leads to a lower quality of life (QOL) [1-4]. VDT use also induces oxidative stress and excessive activation of the autonomic nervous system [5]. Thus, it is very important to improve eyestrain caused by work at VDT.

Rutin is one of antioxidant compounds, and its derivatives are contained in various food plants such as the Japanese pagoda tree (Styphnolobium japonicum, formerly known as Sophora japonica) [6]. Rutin shows antioxidant effects [7] and vasodilatation activity [8]. Monoglucosyl rutin (MGR) is a rutin derivative in which glucose is conjugated to rutin (Fig. 1) [9]. MGR is better dissolved in water than rutin. Previous reports have shown that administration of a rutin derivative (troxerutin) improved eyestrain in a clinical study [10] and that rutin promoted the recovery of retinal function after ischemic insult in rats [11]. These reports suggested that MGR may contribute to the recovery from eyestrain.

Hence, we assumed that MGR could affect eyestrain in humans and investigated its effects on eyestrain in a randomized, double-blind, placebo-controlled, crossover study. We also evaluated the safety of MGR ingestion.

\section{Methods}

\section{Ethics}

This clinical study adhered to the ethical standards of the Declaration of Helsinki of 1964, as modified by subsequent revisions, and to the ethical guidelines for epidemiological research of the Ministry of Education, Culture, Science, and Technology and the Ministry of Health, Labor and Welfare of Japan. The experimental protocol (no. 2016-009) was approved by the Institutional Review Board of Kirin Group Japan Integrated Beverages, Kirin Company, Ltd. This study was registered with the University Hospital Medical Information Network (UMIN) Clinical Trials Registry as UMIN000026566 and was conducted in compliance with the protocol as registered. This clinical trial was conducted from March 2017 to May 2017 in Kirin company Itd, Kanagawa, Japan.

\section{Participants}

Healthy adult males and females aged 20 to 65 years old, who had subjective symptoms of eyestrain, were enrolled, and written informed consent was obtained from each individual. The exclusion criteria were as follows: a possible onset of allergy symptoms; treatment for or a history of drug addiction or alcoholism; any history of a serious disease (e.g., heart disease, respiratory disorder, digestive disturbance, endocrine disorder, metabolic disturbance, or food allergy); constant use of pharmaceuticals for a chronic malady; any history of ophthalmic disease; a surgical history related to a digestive organ; 
donation of more than $200 \mathrm{~mL}$ of blood or blood components within a month prior to this study or over $400 \mathrm{~mL}$ of blood or blood components within three months prior to this study; constant use of pharmaceuticals, dietary supplements, or functional foods affecting eyestrain; excessive alcohol consumption; and possible pregnancy, pregnancy, or lactation. Also excluded participants were those unable to stop drinking on the day before the experimental day; participants or possible participants in another clinical study; those judged ineligible on the experimental day, prior to the beginning of the study, by the site investigators for any reason.

\section{Target sample size}

Based on a preliminary human study, administration of aG-rutin PS 500 mg was expected to reduce HFC1 value by 0.8 with a standard deviation of 1.6 compared to the placebo group. Setting the significance level at $5 \%$ by a paired t-test and the power at 0.95 , the number of subjects required per group was estimated to be 18 . To account for an expected dropout rate of $10 \%$, the number of subjects required per group was estimated to be 20 .

\section{Test food}

We used aG-rutin PS as an MGR-containing food ingredient which was purchased from Toyo Sugar Refining Co.,Ltd (Tokyo, Japan), Ceolus ${ }^{\circledR}$ FD301 as microcrystalline cellulose which was purchased from Asahi Kasei Corporation (Tokyo, Japan), hard gelatin capsule (capsule size 1) which was purchased from Capsugel Japan company. (Kanagawa, Japan). We prepared active and placebo capsules using dark brown capsuls. Table 1 shows prescription of test capsules. There was no significant difference in the appearance and other properties between the active and placebo capsules. MGR was measured by highperformance liquid chromatography using a previously described method [12]. The active capsule contained $188.5 \mathrm{mg}$ of MGR, and the placebo capsule did not contain MGR.

\section{Study design}

The present study was designed as a randomized, double-blind, placebo-controlled, crossover study. The site investigator enrolled the participants. The participants were divided into two groups by stratified randomization using table of random numbers by the assigning controller, and each group was assigned either the active or placebo capsule before the first trial period. Randomization block size was 2 . The assigning controller kept the assignment list in a sealed container until the trial was completed. The participants, investigators, and any persons concerned with the study, excluding the assigning controller, remained blinded.

During the test period, the participants were forbidden to take a pharmaceutical for eyestrain, drink alcohol excessively, vigorously exercise, take food containing ingredients affecting eyestrain, participate 
in another clinical study, and participate in blood donation or sampling.

On the day before the experimental day, the participants were forbidden to have a drink, vigorously exercise, consume food containing food ingredients affecting eyestrain or blood flow, as well as drink and eat after 22:00. The participants were asked to maintain a daily life as usual; keep a diary every day and submit it on the experimental day; contact the site investigator immediately if they felt ill during or after the test period; contact the clinic immediately if they were unable to visit the test room on the experimental day; and keep the test information confidential.

On the experimental day, the participants were forbidden to take food or beverage without water, have VDT such as that on a smartphone or personal computer (PC) working, and smoke.

First, the subjects acclimated to the test room conditions for $20 \mathrm{~min}$. Before the sample intake, we measured the eye status as the pre-intake data $(0 \mathrm{~min})$. Then, the participants took the test food (two capsules) with $100 \mathrm{~mL}$ of water. After the test food intake, the subjects did work at VDT with PC for 30 min and relaxed for $20 \mathrm{~min}$. Eyestrain was measured after relaxing. We evaluated the primary endpoint; high-frequency component 1 (HFC1) as an objective eyestrain parameter, measured by AA-2 accommodometer (Nidec Corp., Tokyo, Japan) according to the manufacturer's instructions [13]. We also evaluated the secondary endpoint; subjective symptoms of eyestrain, dry eye, and sleep, stiffness of the neck and shoulder, and waist stiffness using a visual analog scale (VAS) questionnaire [14], with the left side of VAS showing worse feelings and the right side showing better feelings. The VAS score was calculated as a distance from the left (worst) side. The study protocol is accessed in Kirin company.

\section{Statistical analysis}

Results were calculated as the mean \pm standard deviation using the Ekuseru-Toukei 2010 statistical software (Social Survey Research Information Co., Ltd., Tokyo, Japan). Significance of the differences in HFC1 or VAS between the groups taking the placebo and active compound was estimated using paired $t$ tests.

\section{Results}

\section{Participants}

Participants were recruited in March 2017. Twenty-six individuals were evaluated for the eligibility to participate, and 20 healthy adults were enrolled. Figure 2 is a flow diagram of the study from the assessment until the final analysis. Table 4 provides the information on the participants' background. No participant dropped out of the study, and all underwent analysis for safety evaluation. Two participants were excluded from the efficacy evaluation by the site investigator before the study became un-blinded, in accordance with the exclusion criteria. All, except these excluded participants $(n=18)$, entered the efficacy evaluation (Fig. 2).

\section{Efficacy endpoints}


Table 4 shows the results of objective evaluation. HFC1 in the active group was significantly lower than that in the placebo group for the right eye, dominant eye, and both eyes There were no time effect and carryover effect.

Table 5 shows the results of subjective evaluation. The eyestrain and waist stiffness scores were significantly higher in the active group than those in the placebo group.

\section{Safety endpoint}

There were no adverse events in this study.

\section{Discussion}

We conducted a randomized, placebo-controlled, double-blind, crossover study to evaluate the efficacy and safety of MGR ingestion for eyestrain in healthy people.

VDT causes eyestrain and stiffness of the shoulder and neck, thereby leading to a lower quality of life [14]. Generally, the ciliary muscle in the eye is the key muscle to focus the lens adjustment for clear viewing. VDT causes a high-tension state of the ciliary muscle in the eye, and a continuous stress state of the ciliary muscle causes eyestrain [15]. HFC1 represents the activity of the ciliary muscle. Kajita et al. [13] have reported that the eyestrain caused by VDT use is associated with an increase of the HFC1 value.

In our study, ingestion of MGR lowered HFC1 after VDT work compared to that in the placebo group. Based on this result, we confirmed that MGR provided a rapid recovery from the heavy ciliary muscle contraction caused by VDT use.

MGR is a rutin-glucose conjugate, which is decomposed to rutin or quercetin by digestive enzymes in the small intestine and absorbed as quercetin in the serum [16]. In previous studies, rutin or quercetin showed neuroprotective effects on the rat retina $[17,18]$, retinal function recovery $[11,19]$, and ocular blood flow in animals $[11,20]$. In particular, quercetin improved dry eye symptoms in mice [21]. Based on this evidence, we assumed that MGR is absorbed as quercetin in the serum and modulates the ocular blood flow, especially in the ciliary muscle, and induces the relaxation of the ciliary muscle. Thus, we believe that the mechanism of MGR effects on eyestrain is related to the microvascular blood circulation through nitric oxide (NO) production.

The continuous restricted status of the ciliary muscle constriction causes subjective feelings of the eyestrain, shoulder stiffness, and waist stiffness [22]. In this study, we observed that subjective eyestrain symptoms were improved by MGR administration. Blood flow circulation is related not only to eyestrain but also to waist [1]. We assumed that blood flow modulation by MGR is systemic and that MGR affects not only eyestrain but also waist stiffness.

We confirmed that MGR improved the eyestrain caused by work at VDT, and the results obtained from the VAS questionnaire supported the MGR efficacy for eyestrain. 
In addition, some food ingredients, such as anthocyanins [23-25], crocetin [26, 27], astaxanthin [28, 29], and lutein [30,31], which have antioxidant activities and improve peripheral circulation, have been reported to help recover eyestrain. MGR also has an antioxidant activity and improves peripheral circulation. We believe that the mechanism of MGR involved in the improvement of eyestrain is similar to those of antioxidant chemicals, which does not contradict our results.

In a previous clinical study, a rutin derivative (troxerutin) showed an effect on retinal vein occlusion [10], and multiple rutin-containing food supplements reduced the ocular discomfort [32]. However, there have been no clinical study on the MGR efficacy for eyestrain. We demonstrated for the first time that MGR showed an effect on eyestrain in a clinical study.

In terms of safety evaluation, there were no adverse events in this clinical study, which indicated that MGR-containing food is safe.

A limitation of this study was that it investigated only the MGR efficacy for eyestrain caused by work at VDT on PC. In a future study, we plan to investigate the MGR efficacy for eyestrain caused by other eyestrain situations such as driving.

In general, eyestrain symptom is related to working productivity. Thus, we assume that MGR might have effect on working productivity in workin place.

In general, MGR is one of the safety food ingredient. In this study, we revealed that MGR have effect on eyestrain with VDT work. We strongly believe that MGR is very useful food ingredient for people who are suffered from eyestrain.

\section{Conclusions}

In conclusion, we confirmed that MGR contributed to a rapid recovery from the eyestrain caused by work at VDT. Therefore, MGR is a beneficial substance for people feeling eyestrain.

\section{Abbreviations}

HFC: High-frequency component; MGR: Monoglucosyl rutin; PC: personal computer; QOL: Quality of life; UMIN: University Hospital Medical Information Network; VAS: Visual analog scale; VDT: Visual display terminal.

\section{Declarations}

\section{Acknowledgements}

We thank all volunteers who participated in the study.

\section{Funding}


This study was funded by Kirin Co., Ltd.

\section{Availability of data and materials}

The data that support the findings of this study are available from Kirin company, but restrictions apply to the availability of these data, which were used under license for the current study, and so are not publicly available. Data are however available from the authors upon reasonable request.

\section{Authors' contributions}

Designed the experiment: HA, TK, HF and YK. Performed the experiments: HA, TK, HF and YK. Analyzed the data: HA. Interpreted the results and contributed to the paper: HA and YK. Wrote the manuscript: HA. All authors read and approved the final manuscript.

\section{Ethics approval and consent to participate}

The study protocol was approved by the Institutional Review Board of Kirin Group Japan Integrated Beverages, Kirin Company, Ltd. (Protocol no. 2016-009). Informed consent was obtained from each participant before the commencement of the study.

\section{Consent for publication}

Not applicable.

\section{Competing interests}

HA, TK, HF, and YK are employees of Kirin Co., Ltd., the study sponsor. We certify that there is no conflict of interest with any financial organization regarding the material discussed in the manuscript. The funder designed this study and supplied the test tablets and placebo. The funder had no role in data collection nor data analysis. This manuscript was prepared by the members of the funder. The funder decided to publish the results. The authors declare that they have no competing interests.

\section{References}

1. Parihar JK, Jain VK, Chaturvedi P, Kaushik J, Jain G, Parihar AK. Computer and visual display terminals (VDT) vision syndrome (CVDTS). Med J Armed Forces India. 2016;72: 270-6.

2. Eltayeb S, Staal JB, Hassan A, de Bie RA. Work related risk factors for neck, shoulder and arms complaints: a cohort study among Dutch computer office workers. J Occup Rehabil. 2009; 19:31522.

3. Bergqvist U. Possible health effects of working with VDUs. Br J Ind Med. 1989; 46:217-21.

4. Knave BG, Wibom RI, Voss M, Hedström LD, Bergqvist UO. Work with video display terminals among office employees. I. Subjective symptoms and discomfort. Scand J Work Environ Health. 1985; $11: 457-66$. 
5. Gowrisankaran S, Sheedy JE. Computer vision syndrome: a review. Work. 2015; 52:303-14.

6. Hosseinzadeh $\mathrm{H}$, Nassiri-AsI M. Review of the protective effects of rutin on the metabolic function as an important dietary flavonoid. J Endocrinol Invest. 2014; 37:783-8.

7. Boyle SP, Dobson VL, Duthie SJ, Hinselwood DC, Kyle JA, Collins AR. Bioavailability and efficiency of rutin as an antioxidant: a human supplementation study. Eur J Clin Nutr. 2000; 54:774-82.

8. Fusi F, Saponara S, Pessina F, Gorelli B, Sgaragli G. Effects of quercetin and rutin on vascular preparations: a comparison between mechanical and electrophysiological phenomena. Eur J Nutr. $2003 ; 42: 10-7$.

9. Shimoi K, Shen B, Toyokuni S, Mochizuki R, Furugori M, Kinae N. Protection by alpha G-rutin, a watersoluble antioxidant flavonoid, against renal damage in mice treated with ferric nitrilotriacetate. Jpn J Cancer Res. 1997; 88:453-60.

10. Glacet-Bernard A, Coscas G, Chabanel A, Zourdani A, Lelong F, Samama MM. A randomized, doublemasked study on the treatment of retinal vein occlusion with troxerutin. Am J Ophthalmol. 1994; 118:421-9.

11. Chiou GC, Xu XR. Effects of some natural flavonoids on retinal function recovery after ischemic insult in the rat. $\mathrm{J}$ Ocul Pharmacol Ther. 2004; 20:107-13.

12. Sunada S, Fujisawa H, Cartwright IM, Maeda J, Brents CA, Mizuno K, et al. Monoglucosyl-rutin as a potential radioprotector in mammalian cells. Mol Med Rep. 2014; 10:10-4.

13. Kajita M, Ono M, Suzuki S, Kato K. Accommodative microfluctuation in asthenopia caused by accommodative spasm. Fukushima J Med Sci. 2001; 47:13-20.

14. Kawabata F, Tsuji T. Effects of dietary supplementation with a combination of fish oil, bilberry extract, and lutein on subjective symptoms of asthenopia in humans. Biomed Res. 2011; 32:387-93.

15. Ye Z, Abe Y, Kusano Y, Takamura N, Eida K, Takemoto T, et al. The influence of visual display terminal use on the physical and mental conditions of administrative staff in Japan. J Physiol Anthropol. $2007 ; 26: 69-73$.

16. Matsukawa N, Matsumoto M, Chiji H, Hara H. Oligosaccharide promotes bioavailability of a watersoluble flavonoid glycoside, alpha G-rutin, in rats. J Agric Food Chem. 2009; 57:1498-505.

17. Chang $\mathrm{CH}$, Chiu HF, Han YC, Chen IH, Shen YC, Venkatakrishnan K, et al. Photoprotective effects of cranberry juice and its various fractions against blue light-induced impairment in human retinal pigment epithelial cells. Pharm Biol. 2017; 55:571-80.

18. Ola MS, Ahmed MM, Ahmad R, Abuohashish HM, Al-Rejaie SS, Alhomida AS. Neuroprotective effects of rutin in streptozotocin-induced diabetic rat retina. J Mol Neurosci. 2015; 56:440-8.

19. Arikan S, Ersan I, Karaca T, Kara S, Gencer B, Karaboga I, et al. Quercetin protects the retina by reducing apoptosis due to ischemia-reperfusion injury in a rat model. Arq Bras Oftalmol. 2015; 78:100-4.

20. Park YH, Chiou GC. Structure-activity relationship (SAR) between some natural flavonoids and ocular blood flow in the rabbit. J Ocul Pharmacol Ther. 2004; 20:35-42. 
21. Oh HN, Kim CE, Lee JH, Yang JW. Effects of quercetin in a mouse model of experimental dry eye. Cornea. 2015; 34:1130-6.

22. Faucett J, Rempel D. VDT-related musculoskeletal symptoms: interactions between work posture and psychosocial work factors. Am J Ind Med. 1994; 26:597-612.

23. Uchino Y, Uchino M, Dogru M, Fukagawa $K$, Tsubota K. Improvement of accommodation with antioxidant supplementation in visual display terminal users. J Nutr Health Aging. 2012; 16:478-81.

24. Ozawa Y, Kawashima M, Inoue S, Inagaki E, Suzuki A, Ooe E, et al. Bilberry extract supplementation for preventing eye fatigue in video display terminal workers. J Nutr Health Aging. 2015; 19:548-54.

25. Nakaishi H, Matsumoto H, Tominaga S, Hirayama M. Effects of black current anthocyanoside intake on dark adaptation and VDT work-induced transient refractive alteration in healthy humans. Altern Med Rev. 2000; 5:553-62.

26. Ohno Y, Nakanishi T, Umigai N, Tsuruma K, Shimazawa M, Hara H. Oral administration of crocetin prevents inner retinal damage induced by N-methyl-D-aspartate in mice. Eur J Pharmacol. 2012; 690:84-9.

27. Yamauchi M, Tsuruma K, Imai S, Nakanishi T, Umigai N, Shimazawa M, et al. Crocetin prevents retinal degeneration induced by oxidative and endoplasmic reticulum stresses via inhibition of caspase activity. Eur J Pharmacol. 2011; 650:110-9.

28. Huang JY, Yeh PT, Hou YC. A randomized, double-blind, placebo-controlled study of oral antioxidant supplement therapy in patients with dry eye syndrome. Clin Ophthalmol. 2016; 10:813-

29. Otsuka T, Shimazawa M, Inoue Y, Nakano Y, Ojino K, Izawa H, et al. Astaxanthin protects against retinal damage: evidence from in vivo and in vitro retinal ischemia and reperfusion models. Curr Eye Res. 2016; 41:1465-72.

30. Sawa M, Gomi F, Hara C, Nishida K. Effects of a lutein supplement on the plasma lutein concentration and macular pigment in patients with central serous chorioretinopathy. Invest Ophthalmol Vis Sci. 2014; 55:5238-44.

31. Yagi A, Fujimoto K, Michihiro K, Goh B, Tsi D, Nagai $H$. The effect of lutein supplementation on visual fatigue: a psychophysiological analysis. Appl Ergon. 2009; 40:1047-54.

32. Nebbioso M, Rusciano D, Pucci B, Zicari AM, Grenga R, Pescocolido N. Treatment of glaucomatous patients by means of food supplement to reduce the ocular discomfort: a double blind randomized trial. Eur Rev Med Pharmacol Sci. 2013; 17:1117-22.

\section{Tables}

Table 1 The test capsules (1 capsule)

\begin{tabular}{lcc}
\hline & Placebo & Active \\
\hline aG-rutin PS, mg. & 0 & 250 \\
Microcrystalline cellulose, mg. & 280 & 30 \\
\hline
\end{tabular}


Table 2 nutrient components (2 capsule)

\begin{tabular}{lcc}
\hline & Placebo & Active \\
\hline Energy, kcal. & 0.7 & 2.6 \\
Protein, g. & 0.2 & 0.2 \\
Lipid, g. & 0 & 0 \\
Carbohydrate, g. & 0.5 & 0.5 \\
Sodium, g. & 0.3 & 0.3 \\
Monoglucosyl rutin, mg. & 0 & 352 \\
\hline
\end{tabular}

Table 3 Participants' background $(n=20)$

\begin{tabular}{ll}
\hline Characteristic & All subjects \\
\hline Age, years & $40.3 \pm 9.87$ \\
Body height, cm & $166.89 \pm 7.48$ \\
\hline Body weight, $\mathrm{kg}$ & $62.89 \pm 9.39$ \\
\hline Eyesight (right) & $0.43 \pm 0.47$ \\
\hline Eyesight (left) & $0.38 \pm 0.48$ \\
\hline
\end{tabular}

mean $\pm \mathrm{SD}$

Table 4 Objective evaluation (high-frequency component 1)

\begin{tabular}{|c|c|c|c|}
\hline Site & group & HFC1 value & 95\% Confidence \\
\hline \multirow[t]{2}{*}{ Right eye } & Placebo & $48.20 \pm 7.00$ & $44.96,51.43$ \\
\hline & Active & $44.90 \pm 5.61 *$ & $42.30,47.49$ \\
\hline \multirow[t]{2}{*}{ Left eye } & Placebo & $44.87 \pm 5.57$ & $42.29,47.44$ \\
\hline & Active & $43.29 \pm 5.76$ & $40.62,45.94$ \\
\hline \multirow[t]{2}{*}{ Dominant eye } & Placebo & $46.80 \pm 6.45$ & $43.83,49.78$ \\
\hline & Active & $43.99 \pm 5.85^{*}$ & $41.29,46.69$ \\
\hline \multirow{2}{*}{ Non-dominant eye } & Placebo & $46.26 \pm 6.64$ & $43.19,49.33$ \\
\hline & Active & $44.19 \pm 5.53$ & $41.64,46.75$ \\
\hline \multirow[t]{2}{*}{ Both eyes } & Placebo & $48.20 \pm 6.46$ & $45.21,51.18$ \\
\hline & Active & $44.09 \pm 5.61 * *$ & 41.50, \\
\hline
\end{tabular}

Mean \pm standard deviation $(n=18)$

${ }^{*} p<0.05 ;{ }^{* *} p<0.01$ (vs. placebo), paired $t$-test

Table 5 Subjective evaluation (visual analog scale questionnaire) 


\begin{tabular}{lccc}
\hline Characteristic & group & VAS score & 95\% Confidence interval \\
\hline Eyestrain, mm & Placebo & $4.99 \pm 1.05$ & $4.84,5.91$ \\
& Active & $5.37 \pm 1.16 *$ & $4.50,5.47$ \\
Dry eye, mm & Placebo & $5.36 \pm 1.10$ & $4.85,5.87$ \\
\multirow{2}{*}{ Sleep, mm } & Active & $5.79 \pm 1.33$ & $5.17,6.40$ \\
\multirow{2}{*}{ Stiffness of the neck and shoulder,mm $m$ m } & Placebo & $4.72 \pm 1.19$ & $4.16,6.26$ \\
& Active & $4.74 \pm 1.58$ & $4.01,5.47$ \\
Waist stiffness, mm & Active & $5.47 \pm 1.51$ & $4.56,5.61$ \\
& Placebo & $5.36 \pm 1.13$ & $4.84,5.88$ \\
& Active & $6.06 \pm 1.29 *$ & $5.46,6.65$ \\
\hline
\end{tabular}

Mean \pm standard deviation $(n=18)$

$* p<0.05$ (vs. placebo), paired $t$-test

\section{Figures}

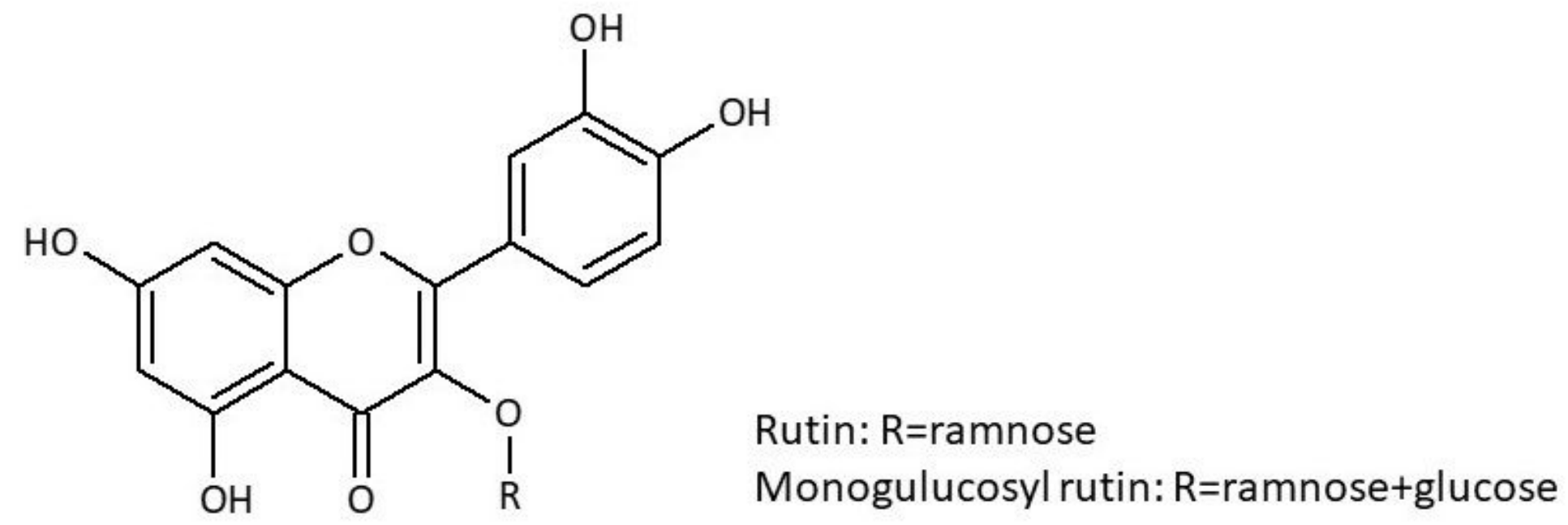

Figure 1

Chemical structures of rutin (a) and monoglucosyl rutin (b) 


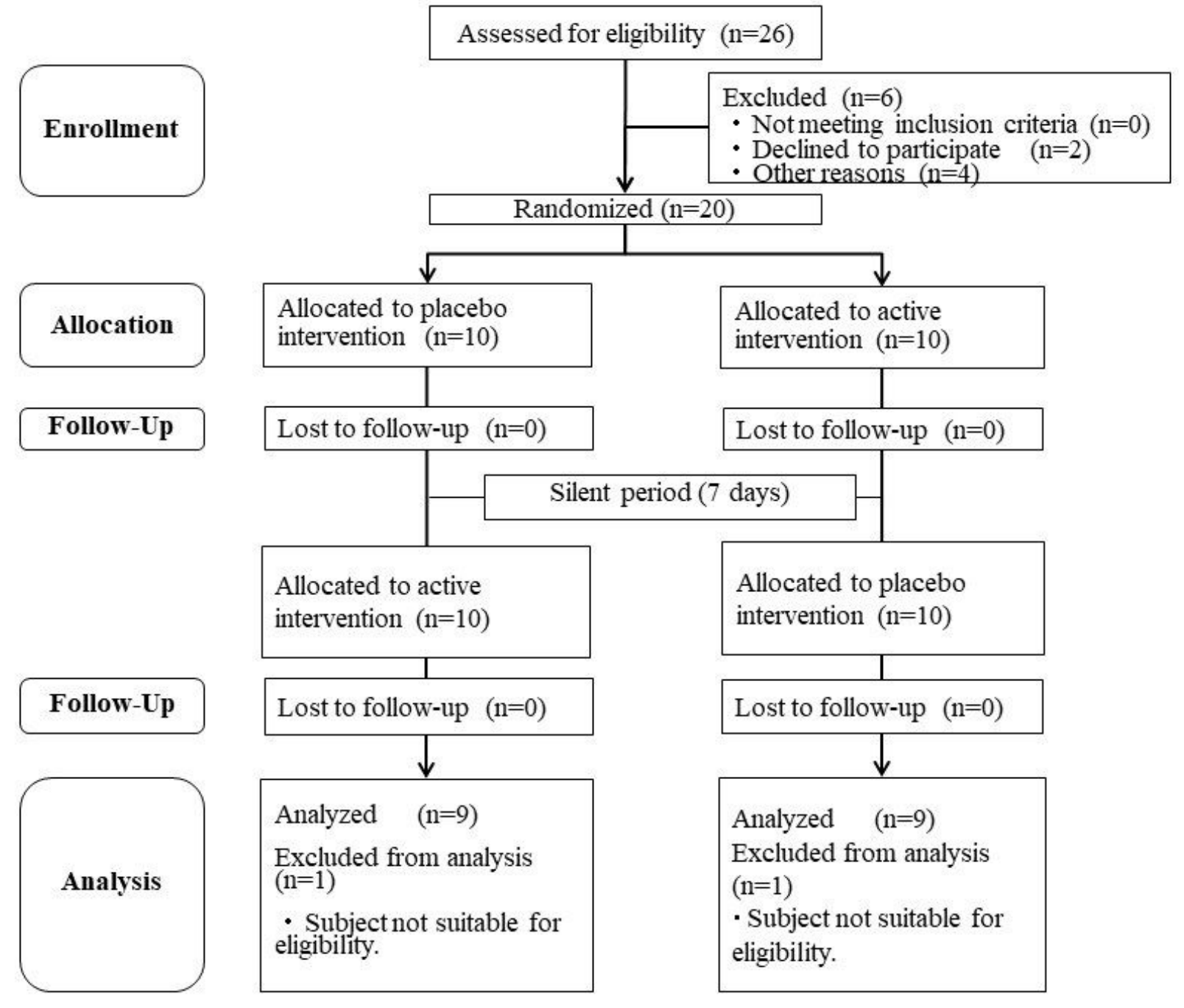

Figure 2

Flow diagram of the progress through the study 